\title{
Looking beyond lesions for causes of neuropsychological impairment in epilepsy
}

Genevieve Rayner, PhD, Chris Tailby, PhD, Graeme Jackson, MD, and Sarah Wilson, PhD

Neurology ${ }^{\circledR}$ 2019;92:e680-e689. doi:10.1212/WNL.0000000000006905
Correspondence

Dr. Rayner

raynerg@unimelb.edu.au

\section{Abstract}

\section{Objective}

Patients with temporal lobe epilepsy (TLE) are similar in their epileptology regardless of whether they have a lesion evident on MRI; this study aims to prospectively clarify whether they are also similar in their neuropsychological profiles.

\section{Methods}

Participants comprised 152 adults: 79 patients with TLE and 73 healthy controls. Patients and controls did not differ in age, sex, or education $(p>0.05)$. Sixty-two percent of patients had an MRI-resolvable lesion (39\% with presumed hippocampal sclerosis [HS-TLE], $61 \%$ with a lesion other than HS [MRI-positive TLE]); the remaining $38 \%$ of patients were lesion-negative. Psychometric measures well established in epilepsy were used.

\section{Results}

Relative to controls, all 3 patient subgroups showed significantly impaired autobiographical, verbal, and visual memory $(p<0.05-0.001)$ and significantly more depression and anxiety $(p<$ $0.05-0.01)$. Yet, contrary to expectations, the 3 TLE subgroups did not differ in their severity of memory or mood impairment $(p>0.05)$. Lower Full-Scale IQpredicted memory impairments across all TLE subtypes, with early age at seizure onset a predictor unique to MRI-negative TLE.

\section{Conclusions}

MRI-negative TLE is associated with memory and mood dysfunction equivalent to that seen in patients with hippocampal sclerosis and other MRI-resolvable pathologies. As such, neuropsychological impairments in TLE are not contingent on a macroscopic lesion and might be an intrinsic property of the underlying network disease.

From the Florey Institute of Neuroscience and Mental Health (G.R., C.T., G.J., S.W.), and Comprehensive Epilepsy Programme, Austin Health (G.J., S.W.), Melbourne Brain Centre, Heidelberg; Melbourne School of Psychological Sciences (G.R., S.W.), the University of Melbourne, Parkville; and Institute for Social Neuroscience (C.T.), Heidelberg, Australia.

Go to Neurology.org/N for full disclosures. Funding information and disclosures deemed relevant by the authors, if any, are provided at the end of the article. 


\section{Glossary}

AMI = Autobiographical Memory Interview; ANCOVA = analysis of covariance; CI $=$ confidence interval; DSM-IV = Diagnostic and Statistical Manual of Mental Disorders (Fourth Edition); ES = effect size; FSIQ = Full-Scale IQ; HS = hippocampal sclerosis; HS-TLE = temporal lobe epilepsy with hippocampal sclerosis; MRI-neg TLE = MRI-negative temporal lobe epilepsy; MRI-pos TLE = MRI-positive temporal lobe epilepsy; NDDI-E = Neurological Disorders Depression Inventory for Epilepsy; PHQ-GAD-7 = Patient Health Questionnaire-Generalized Anxiety Disorder 7-item; ROCF = Rey-Osterrieth Complex Figure; SCID = Structured Clinical Interview for DSM-IV Axis I Disorders; TLE = temporal lobe epilepsy; VPA = Verbal Paired Associates.

A structural brain lesion is detectable on MRI in approximately $65 \%$ of people with epilepsy. ${ }^{1}$ In temporal lobe epilepsy (TLE), the most common pathology is hippocampal sclerosis (HS), ${ }^{2}$ with the study of HS-TLE informing TLE's archetypal neuropsychological profile of anterograde memory impairment. ${ }^{3}$ This led to an inference that memory disturbance in TLE is the result of structural compromise due to "nonfunctional" tissue. ${ }^{4-6}$

Growing evidence, however, indicates that memory deficits in TLE can occur on a background of diffuse cognitive disturbances that implicate regions beyond the epileptogenic zone. ${ }^{7}$ This fits with our evolving understanding of epilepsy as a disease that propagates and alters large-scale networks subserving cognitive and emotional processing, ${ }^{8}$ whereby any etiology that results in epileptogenic disease may undermine neuropsychological function. ${ }^{9-11}$ This conceptual shift questions the specific effects of a lesion on neuropsychological functioning in TLE, as opposed to the effects of underlying network disease common to all cases. To date, studies comparing memory in MRI-negative TLE to lesional cases have been low-powered, ${ }^{5,12}$ failed to provide appropriate comparison groups, ${ }^{12}$ and report mixed findings. $5,13,14$

We aimed to prospectively clarify the contribution of macroscopic structural compromise to neuropsychological dysfunction in TLE. We hypothesized that patients with MRInegative TLE will perform worse than controls on measures of memory and mood. The cumulative effect of a lesion occurring in addition to network disease, however, will give rise to even greater neuropsychological impairment; i.e., HS-TLE and MRI-positive TLE will perform worse than MRI-negative cases. Finally, we delineate predictors of neuropsychological dysfunction in these groups.

\section{Methods}

\section{Participants}

Inclusion criteria for all participants $(\mathrm{n}=152)$ were $(1)$ age 18-70 y, (2) Full-Scale IQ (FSIQ) score $\geq 70$ or FSIQ judged to be unimpaired via formal neuropsychological assessment, (3) no history of neurosurgery, and (4) functional English. FSIQ was estimated using the Test of Premorbid Functioning. ${ }^{15}$ Inclusion criteria related to FSIQ pertain to the standard ethics approvals granted to this study, in which adults with a known or likely intellectual disability are deemed unable to give independent informed consent to participate in research. Moreover, the validity of the measures of memory and mood administered to the sample depends on the participants' ability to read and comprehend self-report measures, recognize and convey abstract mood symptomatology, as well as follow complex task instructions. The variability of persons with intellectual disability to conform to these standards was seen to introduce an unacceptable level of confound to study design. ${ }^{16}$

Exclusion criteria for patients comprised a history of neurologic disease other than epilepsy, and psychiatric diagnoses other than depressive or anxiety disorders. Exclusion criteria for the controls comprised any history of neurologic disease or major psychiatric illness.

\section{Standard protocol approvals, registrations, and patient consents}

In accordance with the Declaration of Helsinki, this study was approved by the relevant institutional human research ethics committees, and all participants provided written, informed consent.

\section{Patients}

The patient sample $(\mathrm{n}=79)$ was prospectively recruited from the Comprehensive Epilepsy Program of Austin Health, Melbourne. Epileptogenic foci were localized to the temporal lobe by methods previously published by our group, ${ }^{17}$ including clinical history, semiology, video$\mathrm{EEG}$, 3-tesla MRI, interictal $\left[{ }^{18} \mathrm{~F}\right]$ fluorodeoxyglucosePET, ictal SPECT, and neuropsychological evaluation. Demographic and clinical features of the TLE sample are summarized in table 1 . The proximity of the last seizure to neuropsychological testing was not routinely recorded.

Nineteen patients (24\%) had a focal lesion detectable on clinical MRI consistent with HS-TLE, 30 (38\%) had a focal lesion confined to the temporal lobe judged to be an epileptogenic pathology other than HS (MRI-pos TLE), and $30(38 \%)$ did not have any focal lesion detectable on MRI (MRI-neg TLE; table 1). Key subgroup clinical findings were: 
Table 1 Demographic and clinical profile of temporal lobe epilepsy subgroups and healthy controls

\begin{tabular}{|c|c|c|c|c|}
\hline & MRI-neg TLE $(n=30)$ & HS-TLE ( $n=19)$ & MRI-pos TLE $(n=30)^{a}$ & Healthy controls $(n=73)$ \\
\hline Age, $y$, mean \pm SD (range) & $35.27 \pm 9.86(23-57)$ & $42.05 \pm 12.67(23-66)$ & $38.27 \pm 11.54(20-57)$ & $34.33 \pm 14.33(18-59)$ \\
\hline Sex, F, n (\%) & $18(60)$ & $14(74)$ & $13(43)$ & $53(73)$ \\
\hline Education, $y$, mean $\pm S D$ (range) & $14.62 \pm 3.72(10-24)$ & $13.21 \pm 3.42(9-22)$ & $13.58 \pm 3.43(8-24)$ & $14.29 \pm 2.62(9-21)$ \\
\hline Full-Scale IQ, mean \pm SD (range) & $102.03 \pm 11.90(81-130)$ & $101.89 \pm 13.10(75-132)$ & $99.62 \pm 9.05^{a}(72-120)$ & $105.85 \pm 11.97^{\mathrm{b}, \mathrm{f}}(71-132)$ \\
\hline $\begin{array}{l}\text { Age at seizure onset, } y \text {, mean } \pm \text { SD } \\
\text { (range) }\end{array}$ & $21.24 \pm 10.98(2-56)$ & $19.05 \pm 13.75(2-51)$ & $25.11 \pm 14.61(0.3-50)$ & - \\
\hline $\begin{array}{l}\text { Duration of epilepsy, } y \text {, mean } \pm \text { SD } \\
\text { (range) }\end{array}$ & $14.57 \pm 9.85(2-37)$ & $23.37 \pm 17.23(3-62)$ & $13.27 \pm 9.24(2-37)$ & - \\
\hline $\begin{array}{l}\text { Monthly seizure frequency, mean } \pm S D \text {; } \\
\text { median (range) }\end{array}$ & $8.93 \pm 11.58 ; 5.00(1-50)$ & $8.95 \pm 14.84 ; 4.00(1-67)$ & $23.73 \pm 45.89 ; 4.50(1-200)$ & - \\
\hline \multicolumn{5}{|l|}{ Side of epilepsy focus, $n$ (\%) } \\
\hline Left & $17(57)$ & $7(38)$ & $14(47)$ & - \\
\hline Right & $12(40)$ & $9(47)$ & $16(53)$ & - \\
\hline Bilateral/unclear & $1(3)$ & $3(16)$ & 0 & - \\
\hline PET-concordant, $n(\%)^{c}$ & $16(64)^{d, g}$ & $15(100)^{e}$ & $23(92)^{d}$ & - \\
\hline Febrile convulsions, n (\%) & $2(7)$ & $8(42)^{g}$ & $5(17)$ & - \\
\hline History status epilepticus, n (\%) & 0 & $3(16)^{f}$ & $1(3)$ & - \\
\hline History of encephalopathy, n (\%) & 0 & $2(11)^{f}$ & 0 & - \\
\hline Surgical candidate, n (\%) & $4(13)^{\mathrm{h}}$ & $14(74)$ & $27(90)$ & - \\
\hline Antiepileptic drug polytherapy, $n$ (\%) & $22(74)$ & $14(74)$ & $22(74)$ & - \\
\hline $\begin{array}{l}\text { No. of antiepileptic drugs, mean } \pm \text { SD } \\
\text { (range) }\end{array}$ & $2.13 \pm 1.01(1-4)$ & $2.16 \pm 1.07(0-4)$ & $2.10 \pm 0.86(1-4)$ & - \\
\hline
\end{tabular}

Abbreviations: HS-TLE = temporal lobe epilepsy with hippocampal sclerosis; MRI-neg TLE = MRI-negative temporal lobe epilepsy; MRI-pos TLE = MRI-positive temporal lobe epilepsy.

a Ten cases of WHO grade I tumor, including dysembryoplastic neuroepithelial tumors, 5 cases of dysplasia, 3 cases of encephalocele, 2 cases of cavernoma, 2 cases of tuberous sclerosis, and 1 case each of WHO grade II tumor, trauma, polymicrogyria, and heterotopia.

b One case of missing data.

ci.e., a PET abnormality that is congruent with the focality and laterality of the seizure focus according to video-EEG/semiology.

${ }^{\mathrm{d}}$ Five cases of missing data.

e Four cases of missing data.

${ }^{f} p<0.05$.

${ }^{\mathrm{g}} p<0.01$.

${ }^{\mathrm{h}} p<0.001$.

\section{HS-TLE}

- more likely to have had febrile convulsions (42\%) relative to MRI-pos $(17 \%)$ and MRI-neg TLE $\left(7 \% ; \chi_{2}^{2}=\right.$ 9.67, $p=0.008, \phi=0.35$, medium effect size [ES]);

- more likely to have experienced status epilepticus (16\%) relative to MRI-pos (3\%) and MRI-neg TLE (0\%; $\chi_{2}^{2}=6.21, p=0.045, \phi=0.28$, medium ES);

- more likely to have had encephalitis (11\%) relative to MRI-pos and MRI-neg TLE, none of whom had had it $\left(\chi_{2}^{2}=6.48, p=0.039, \phi=0.29\right.$, medium ES $)$.

2. MRI-neg TLE

- less likely to be deemed surgical candidates (13\%) compared to HS-TLE (74\%) and MRI-pos TLE (90\%; $\chi_{2}^{2}=35.60, p<0.001, \phi=0.69$, large ES];

- less likely to have a concordant PET abnormality (64\%) vs HS-TLE (100\%) and MRI-pos TLE (92\%; $\chi_{2}^{2}=10.94, p=0.004, \phi=0.41$, medium to large ES); unlike in previous studies, $\left[{ }^{18} \mathrm{~F}\right]$ fluorodeoxyglucosePET concordance encompassed focalization to the temporal lobe in addition to the putative laterality and might account for the lower rates of PET concordance in MRI-negative compared to previous studies ${ }^{12}$;

- when PET-concordant, did not differ from those who did not have a concordant PET abnormality in terms of epileptologic features, such as age at onset, seizure frequency, and lateralization $(p<0.05$ for all comparisons).

\section{Healthy controls}

Seventy-three healthy individuals were recruited from the patients' families and broader community to provide a sociodemographically matched control sample of similar size to the 
patient sample. Patients and controls did not differ in sex $\left(\chi^{2}=\right.$ $3.40, p=0.065)$, age $\left(t_{137,33}=1.76, p=0.081\right)$, or years of education $\left(t_{150}=-8.15, p=0.42\right.$; table 1$)$. Controls had a slightly higher FSIQ than the patients overall $\left(t_{148}=-2.51\right.$, $p=0.013, \eta^{2}=0.04$, small ES); however, mean scores for both groups fell within the "average" range (90-110).

\section{Assessments}

\section{Neuropsychiatric evaluation}

In-depth neuropsychiatric evaluation of patients was undertaken using the Structured Clinical Interview for DSM-IV Axis I Disorders (SCID), the gold standard for diagnosing mood disturbance according to the criteria of the DSM-IV. ${ }^{18}$ The SCID includes questioning around atypical symptoms of depression some researchers consider to be of especial relevance to epilepsy. ${ }^{19}$ Patients were carefully questioned about depressive symptoms to ensure they could not be directly attributed to changes in antiepileptic medication.

The Neurological Disorders Depression Inventory for Epilepsy (NDDI-E) ${ }^{20}$ assesses the frequency of 6 current depressive symptoms, which do not overlap with commonly comorbid cognitive deficits in epilepsy or the adverse effects of antiepileptic drugs. NDDI-E total scores range from 6 to 28 ; scores $>15$ have $90 \%$ specificity and $81 \%$ sensitivity for a diagnosis of major depression.

The Patient Health Questionnaire-Generalized Anxiety Disorder 7-item (PHQ-GAD-7) assesses the severity of 7 current anxiety symptoms in medical populations. ${ }^{21}$ Participants assign scores of 0 to 3 to the response categories of "not at all" through to "nearly every day"; total scores range from 0 to 21 , and scores of 5,10 , and 15 represent cutoffs for mild, moderate, and severe anxiety, respectively.

\section{Memory assessment}

The Autobiographical Memory Interview (AMI) ${ }^{22}$ assesses recall of personal memories from childhood, early adulthood, and recent life. The Personal Semantic Schedule requires participants to recollect personally relevant facts across the 3 time points (e.g., former addresses; maximum score $=63$ ), with scores $\leq 47$ associated with an amnestic syndrome and 48 or 49 a probable amnestic syndrome. The Autobiographical Incidents Schedule asks participants to recall 3 episodes from each time period (e.g., a wedding ceremony). Episodic memories are scored from 0 to 3 (maximum $=27$ ) based on their richness in detail and how precisely the incident is located in place and time, with total scores $\leq 12$ associated with an amnestic syndrome, and 13 to 15 a probable amnestic syndrome. Interrater reliability is $r=0.83-0.86$, with good sensitivity to organic disease.

Auditory-verbal memory was assessed using the Wechsler Memory Scale-Fourth Edition ${ }^{23}$-specifically, immediate and delayed recall indices of the Verbal Paired Associates (VPA) subtest. The examiner presents a list of 14 word pairs; the participant then hears one word and must provide the word that went with it. Some pairs are semantically related (e.g., "street-road"), considered an indicator of lateral temporal lobe integrity; others are arbitrarily associated (e.g., "bed-lost"), considered an indicator of mesial temporal lobe integrity. $3,23,24$ There are 4 learning trials and one delayed recall trial, scored according to age-scaled normative data $($ mean $=10 ; \mathrm{SD}=3)$; i.e., scaled scores $<8$ are indicative of below-average performance.

The delay trial of the Rey-Osterrieth Complex Figure (ROCF) is a widely used test of figural memory retrieval. ${ }^{25}$ The participant must copy the figure, reproducing it from memory 30 minutes later. The figure was scored according to the system proposed by Osterrieth that reduces it to 18 details that are scored 2, 1, or 0.5 (maximum score $=36$ ). It has high inter- and intrarater reliability $(>0.8)$ and internal consistency $(>0.6)$.

\section{Data availability}

Anonymized data will be shared by request from any qualified investigator.

\section{Statistical analyses}

Analyses were performed using IBM SPSS Statistics (IBM Corp., Armonk, NY) and R (R Foundation for Statistical Computing, Vienna, Austria). The Benjamini-Hochberg procedure was used throughout to control the false discovery rate stemming from multiple comparisons, ${ }^{26}$ with significance set at $p<0.05$ (2-tailed). Where data did not meet assumptions for parametric analyses, conservative alternatives were used (e.g., Mann-Whitney U, Brown-Forsythe). Some control data were missing because of a change in protocol, so Little's missing completely at random test was used on a subset of cases and showed that there was no relationship between the missingness of data and any values $\left(\chi_{2}^{2}=4.212\right.$, $p>0.050)$. Very minimal patient data were missing and typically occurred because of interruptions in testing by seizures or early discharge. Given the difference in FSIQ between patients and controls, scatterplots and Pearson correlations were used to assess the relationships between memory indices and FSIQ in these 2 groups; no significant relationships were identified $(r<0.3)$, negating the need to covary for FSIQ in subsequent analyses.

To test the hypothesis that the neuropsychological performances in the MRI-neg TLE group would be worse than those of controls, their memory and mood scores were compared using $\chi^{2}$ analyses for categorical variables and independentsample $t$ tests for continuous variables (illustrated by the figure).

To test the hypothesis that patients with either HS-TLE or MRI-pos TLE would score worse than patients with MRI-neg TLE on measures of mood and memory, 1-way betweensubjects analysis of variance with planned contrasts were used (illustrated by the figure). The effect of seizure laterality on 

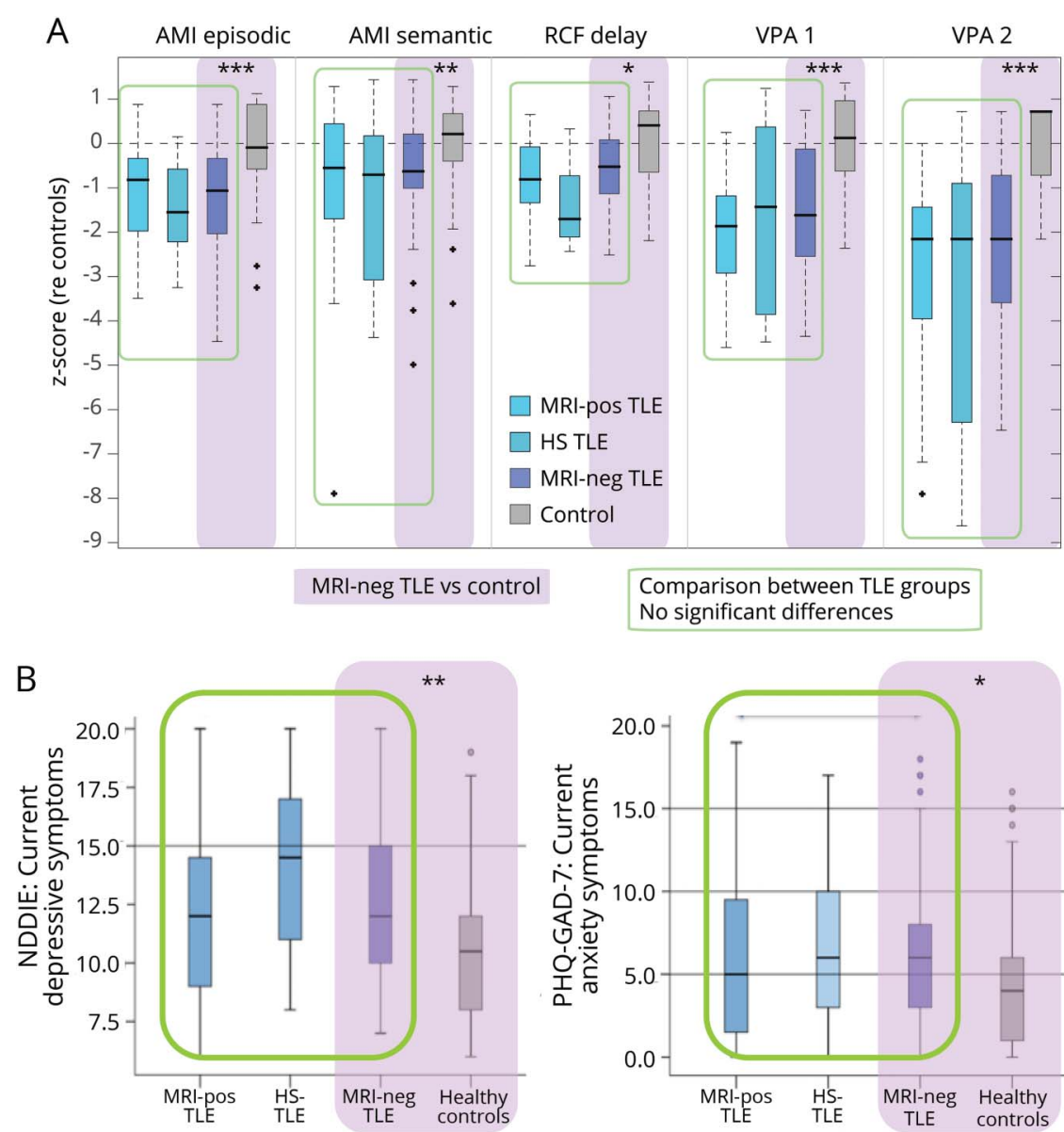

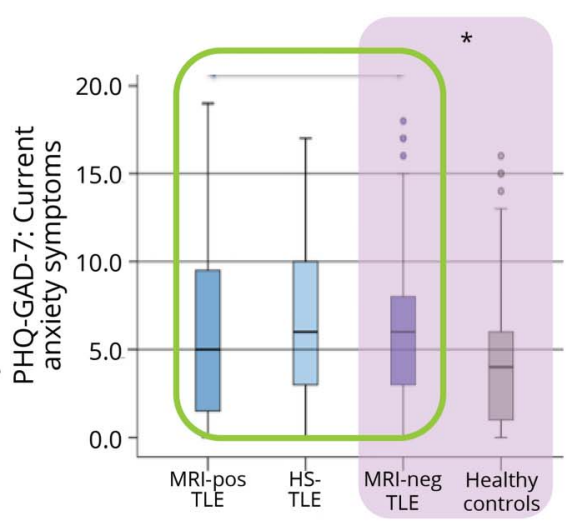

Relative to controls, patients with MRI-neg TLE showed significantly impaired autobiographical, verbal, and visual memory $(A)$, as well as significantly more symptoms of depression and anxiety (B). Contrary to expectations, the 3 TLE subgroups showed no significant differences in memory (A) or mood (B). These boxplots represent $z$ scores calculated from the healthy control raw data. Purple shading and asterisks delineate comparisons between the MRI-neg TLE group and healthy controls, while green boxes delineate comparisons between the 3 TLE groups (no significant differences on any measure). ${ }^{*} p<0.05$; $\star * p<0.01 ; * \star \star p p<0.001$. AMI = Autobiographical Memory Interview; HS-TLE = temporal lobe epilepsy with hippocampal sclerosis; MRI-neg TLE = MRI-negative temporal lobe epilepsy; MRI-pos TLE = MRI-positive temporal lobe epilepsy; NDDI$\mathrm{E}=$ Neurological Disorders Depression Inventory for Epilepsy; PHQ-GAD-7 = Patient Health Questionnaire-Generalized Anxiety Disorder 7item; RCF = Rey Complex Figure; VPA = Verbal Paired Associates. neuropsychological functioning in the 3 TLE groups was assessed with nonparametric statistics and was found to be nonsignificant $(p>0.05)$, discounting the need for covariance in subsequent analyses.

Finally, exploratory analyses examined the differential predictors of memory dysfunction in TLE subgroups using analysis of covariance (ANCOVA). The number of outcome variables was reduced by selecting one from each domain (autobiographical, verbal, visual memory), choosing the variable with the largest ES when comparing MRIneg TLE to controls: that is, AMI episodic recall, VPA-II, and ROCF delayed recall. The ANCOVA models were used to examine predictors of dysfunction separately for each domain, while accounting for lesion status (3 levels: MRIneg, MRI-pos, HS-TLE). The relative importance of all epilepsy, demographic, and mood predictors (i.e., all those detailed in tables 1 and 2) to these 3 outcome variables was examined as follows: in the case of categorical predictors (e.g., sex), the strength of their relationship with an outcome variable was assessed via analysis of variance or $t$ tests; for linear variables (e.g., seizure frequency), bivariate correlations. Only independent variables that were significantly associated with the outcome variable were then entered into an ANCOVA model containing the grouping variable "TLEtype." The "step" function in R was used to select the bestfitting model.

For AMI, the initial model (in R notation) passed to "step" was specified as:

$$
\text { AMI TLEtype } \times \text { Onset }
$$

where onset $=$ age at onset.

For VPA-II, the initial model was specified as:

$$
\text { VPA_II } \sim \text { TLEtype } \times \text { FSIQ }
$$

For ROCF, the initial model was specified as:

$$
\text { RCFT_delay } \sim \text { TLEtype } \times \text { FSIQ }+ \text { TLEtype } \times \text { SCID_pastdep }
$$

where SCID_pastdep = history of depression, as assessed via the SCID.

Significant interactions were followed up with planned contrasts evaluating the significance of the slope parameter $(\beta)$ relating the independent variable to the outcome variable in 
Table 2 Neuropsychological dysfunction in MRI-neg TLE relative to healthy controls $(n=91)$

\begin{tabular}{|c|c|c|c|}
\hline & MRI-neg TLE $(n=30)$ & Healthy controls $(n=61)$ & Effect size, Cohen $d$ \\
\hline \multicolumn{4}{|c|}{ Autobiographical memory } \\
\hline AMI semantic recall & $55.97 \pm 4.73(42-63)$ & $58.30 \pm 3.27^{f}(57-62.5)$ & 0.62 (medium) \\
\hline AMI episodic recall & $17.20 \pm 5.05(4-25)$ & $22.38 \pm 4.12^{g}(24-27)$ & 1.18 (very large) \\
\hline \multicolumn{4}{|c|}{ Auditory-verbal learning and recall } \\
\hline VPA-I SS & $9.25 \pm 3.27^{b}(4-15)$ & $12.11 \pm 2.867^{\mathrm{d}, \mathrm{g}}(7-17)$ & 0.95 (large) \\
\hline VPA-II SS & $9.00 \pm 2.68^{\mathrm{b}}(5-14)$ & $11.91 \pm 1.809^{\mathrm{d}, \mathrm{g}}(10-19)$ & 1.317 (very large) \\
\hline \multicolumn{4}{|l|}{ Visuospatial recall } \\
\hline ROCF delayed recall & $21.52 \pm 5.44^{a}(7-15)$ & $25.48 \pm 6.15^{\mathrm{d}, \mathrm{e}}(7-16)$ & 0.69 (medium) \\
\hline \multicolumn{4}{|l|}{ Mood } \\
\hline NDDI-E total & $12.90 \pm 3.78(7-20)$ & $10.80 \pm 3.20^{f}(6-18)$ & 0.63 (medium) \\
\hline PHQ-GAD-7 total & $6.79 \pm 5.49(0-18)$ & $4.37 \pm 4.04^{\mathrm{e}}(0-10)$ & 0.54 (medium) \\
\hline \multicolumn{4}{|c|}{$\begin{array}{l}\text { Abbreviations: AMI = Autobiographical Memory Interview; MRI-neg TLE = MRI-negative temporal lobe epilepsy; NDDI-E = Neurological Disorders Depression } \\
\text { Inventory in Epilepsy; PHQ-GAD-7 = Patient Health Questionnaire-Generalized Anxiety Disorder 7-item; ROCF = Rey-Osterrieth Complex Figure; SS = scaled } \\
\text { score (i.e., mean = } 10 \pm 3 \text { ); VPA = Verbal Paired Associates. } \\
\text { Data represent mean } \pm \text { SD (range). } \\
\text { a Four cases of missing data. } \\
\text { b Two cases missing data. } \\
\text { c Thirty-one missing cases. } \\
\text { d Twenty-six cases of missing data. } \\
\text { e } p<0.05 \text {. } \\
{ }_{f} p<0.01 . \\
\text { g } p<0.001 .\end{array}$} \\
\hline
\end{tabular}

the MRI-neg TLE group, and comparing the slope parameter in the MRI-neg TLE group to those in the HS-TLE group and the MRI-pos TLE group.

\section{Results}

\section{MRI-neg TLE is not neuropsychologically benign}

\section{MRI-neg TLE is not cognitively benign}

As hypothesized, patients with MRI-neg TLE presented with neuropsychological impairment. Relative to controls, they showed impoverished autobiographical, verbal, and visual memory, as well as higher symptoms of depression and anxiety (table 2 for descriptive data and ES). Specifically, they were poorer at recollecting semantic autobiographical details $\left(t_{89}=-2.75, p<0.01,95 \%\right.$ confidence interval $[\mathrm{CI}]-4.02$ to $-0.65)$, episodic autobiographical events $\left(t_{89}=-5.22, p<\right.$ $0.001,95 \%$ CI -7.15 to -3.20 ), visuospatial information on the ROCF after a delay $\left(t_{54}=-2.54, p<0.05,95 \% \mathrm{CI}-7.10\right.$ to $-0.83)$, and were worse at both immediate recall on VPA-I $\left(t_{61}=-3.70, p<0.001,95 \% \mathrm{CI}-4.41\right.$ to -1.32$)$ and later spontaneous retrieval of word pairs on VPA-II (auditoryverbal memory; $t_{46.09}=-4.88, p<0.001,95 \% \mathrm{CI}-4.11$ to -1.71) (figure, A).

Within the MRI-neg group, there was a difference in the episodic autobiographical recollection of those who were
PET-positive and those who were not; namely, those who were lesion-negative and PET-negative were poorer at recalling personal life events $\left(t_{23}=2.28, p=0.033\right.$, 95\% CI 0.37-7.76; Cohen $d=0.99$, i.e., large ES). There were no differences on any other neuropsychological measure between those with and without a congruent PET $(p>0.05)$, or any differences based on seizure onset laterality.

\section{MRI-neg TLE is not psychiatrically benign}

People with MRI-neg TLE endorsed higher levels of depressive symptomatology on the NDDI-E than controls $\left(t_{98}=\right.$ 2.84, $p=0.005,95 \%$ CI 0.63-3.56), as well as higher levels of anxiety-related symptomatology on the PHQ-GAD-7 ( $t_{95}=$ 2.42, $p=0.017$, 95\% CI 0.44-4.41; figure, B). In MRI-neg TLE, depression and anxiety symptoms were strongly correlated $(r=0.89, p<0.001)$.

The SCID revealed that 9 individuals with MRI-neg TLE (30\%) met criteria for a current depressive disorder, higher than the global point prevalence of $4.7 \%$ (95\% CI $4.4 \%-5.0 \%{ }^{27}$; Fisher exact test, $\left.p=0.007\right)$. Fifteen patients with MRI-neg TLE (50\%) had a lifetime history of, or current, mood disorder, markedly higher than $17 \%$ of Australians from the broader community who will experience depression in their lifetime. ${ }^{28}$ There were no laterality or PET effects on mood evident within MRI-neg TLE $(p>0.05)$. 


\section{Comparable neuropsychological dysfunction in MRI-neg and lesional TLE}

Contrary to expectations, patients with MRI-neg TLE performed just as poorly as patients with HS-TLE and MRI-pos TLE across all measures of memory; that is, there were no differences in performance for the 3 groups on any of the psychometric measures $(p>0.05$; table 3 and the figure). There were also no laterality effects evident in the scores of the 3 TLE subtypes.

Similarly, the level of depressive and anxious symptomatology did not differ between groups ( $p>0.05$; table 3 and the figure).

\section{Predictors of memory dysfunction in TLE}

The final model of predictors of episodic autobiographical retrieval (AMI total score, Autobiographical Incidents Schedule) revealed an interaction between TLE type and age at onset $\left(F_{2,69}=3.51, p=0.035\right)$. This interaction reflected better AMI scores with increasing age at onset in the MRI-neg TLE group ( $\beta=0.22, t=2.73, p=0.024)$, with the slope of this relationship greater in MRI-neg TLE than that in MRIpos TLE $(t=-2.25, p=0.028)$ and HS-TLE $(t=-2.42$, $p=0.027)$.
The final model of predictors of auditory verbal memory (VPA-II $)$ included only FSIQ $\left(F_{1,61}=12.0, p=0.001\right)$. VPAII scaled scores increased with increasing FSIQ $(\beta=0.12)$; the influence of FSIQ did not differ between TLE groups.

The final model of predictors of visual recall (ROCF) included TLE type, FSIQ SCID past depression, and a TLE type $\times$ SCID past depression interaction. ROCF scores increased with in creasing FSIQ $\left(\beta=0.06 ; F_{1,58}=5.60, p=0.021\right)$; the influence of FSIQ did not differ between TLE groups.

\section{Discussion}

This study confirmed that neuropsychological dysfunction in epilepsy can arise in the context of macroscopically normal brain tissue, with mood and memory significantly impaired in a large sample of patients with MRI-negative TLE relative to healthy controls. That is, a "normal" MRI in epilepsy is not necessarily neuropsychologically benign. Contrary to expectations, moreover, MRI-negative TLE presented with neuropsychological decrements comparable in magnitude to those seen in HS or other MRI-resolvable lesions. This

Table 3 Comparable neuropsychological dysfunction across temporal lobe epilepsy subtypes ${ }^{a}$

\begin{tabular}{|c|c|c|c|}
\hline & MRI-neg TLE (n = 30) & HS-TLE $(n=19)$ & MRI-pos TLE $(n=30)$ \\
\hline \multicolumn{4}{|l|}{ Autobiographical memory } \\
\hline AMI semantic recall & $55.97 \pm 4.73(42-63)$ & $54.66 \pm 6.13(44-63)$ & $55.44 \pm 6.16(32.5-62.5)^{b}$ \\
\hline AMI episodic recall & $17.20 \pm 5.05(4-26)$ & $15.79 \pm 4.01(9-23)$ & $17.48 \pm 5.06(8-26)^{b}$ \\
\hline \multicolumn{4}{|c|}{ Auditory-verbal learning and recall } \\
\hline VPA-I scaled score & $9.25 \pm 3.27(4-15)^{d}$ & $9.50 \pm 4.98(3-17)^{g}$ & $8.38 \pm 2.60(3-12)^{i}$ \\
\hline VPA-II scaled score & $9.00 \pm 2.68(5-14)^{d}$ & $8.36 \pm 4.43(1-14)^{g}$ & $8.25 \pm 3.12(1-13)^{i}$ \\
\hline \multicolumn{4}{|l|}{ Visuospatial recall } \\
\hline ROCF delayed recall & $11.04 \pm 2.46(7-15)^{e}$ & $10.07 \pm 2.40(6-15)^{f}$ & $10.93 \pm 2.50(6-15)^{b}$ \\
\hline \multicolumn{4}{|l|}{ Mood } \\
\hline NDDI-E total score & $12.90 \pm 3.78(7-20)^{c}$ & $14.00 \pm 3.71(8-20)$ & $11.81 \pm 3.54(6-20)^{b}$ \\
\hline PHQ-GAD-7 total score & $6.79 \pm 5.49(0-18)^{c}$ & $6.88 \pm 5.37(0-17)^{d}$ & $6.22 \pm 5.60(0-19)^{b}$ \\
\hline \multicolumn{4}{|l|}{ Diagnosis of depression } \\
\hline Current depression $^{i}$ & $9(30)$ & $3(16)$ & $6(21)^{d}$ \\
\hline Past depression ${ }^{i}$ & $13(43)$ & $9(47)$ & $6(21)^{d}$ \\
\hline \multicolumn{4}{|c|}{$\begin{array}{l}\text { Abbreviations: AMI = Autobiographical Memory Interview; MRI-neg TLE = MRI-negative te } \\
=\text { = Neurological Disorders Depression Inventory in Epilepsy; PHQ-GAD-7 = Patient Healt } \\
\text { Osterrieth Complex Figure; VPA = Verbal Paired Associates. } \\
\text { Data represent mean } \pm \text { SD (range) or } n(\%) \text {. } \\
\text { a p }>0.05 \text { for all reported variables, based on univariate between-group comparisons. } \\
\text { b Three cases of missing data. } \\
\text { c One case of missing data. } \\
\text { d Two cases of missing data. } \\
\text { e Four cases of missing data. } \\
\text { f Five cases of missing data. } \\
\text { g Eight cases of missing data. } \\
\text { h Six cases of missing data. }\end{array}$} \\
\hline
\end{tabular}


suggests that at the group level, network disease per se is capable of producing memory and affective disturbances as extensive as those produced by overt focal pathology. In MRInegative disease, neuropsychological impairment was specifically linked to earlier age at seizure onset, while lower FSIQ was revealed as a predictor of poor memory across TLE subtypes. Taken together, the findings suggest that neuropsychological impairments in TLE are not contingent on macroscopic lesions, and in some cases, might be conceptualized as an intrinsic property of an underlying network disease with various etiologies.

In line with previous studies, the current findings demonstrate that people with MRI-negative TLE have impaired memory, ${ }^{5,13,14}$ including the personally meaningful domain of autobiographical memory. Moreover, MRI-negative TLE is linked to higher rates of depressive disorder and symptomatology than is seen in controls. Neuropsychological compromise occurring in the absence of a resolvable lesion suggests that macroscopic pathology cannot account for every case of impairment in TLE. Moreover, our results indicate that neuropsychological deficits do not necessarily stem from abnormal hypometabolism, a surrogate marker of microstructural abnormalities not seen on MRI. Without histopathologic analysis, the possibility that a minority of the patients with MRI-negative TLE had abnormal microcellular anatomy contributing to their poor memory cannot be ruled out. ${ }^{5}$ However, it is reasonable to speculate that for the majority of MRI-negative cases, cognitive and affective comorbidities are underpinned by its fundamentally abnormal network function and topography. ${ }^{29,30}$

The findings extend previous work by revealing that at a group level, neither HS nor any other lesional pathology is more pernicious to memory and mood than nonlesional network disease. Inspection of the psychometric results shows that there is considerable heterogeneity within each TLE subtype, with performances of patients with the same lesion status ranging from intact to markedly impaired. That is, while tissue damage and network disease can undermine neuropsychological function in some cases, in others the diseased brain retains sufficient functional integrity to support normal neuropsychological function or perhaps has reorganized to support it. ${ }^{4}$ Lesion status cannot, therefore, be used as a heuristic in predicting cognitive or affective dysfunction in TLE; it remains to be ascertained by careful clinical and psychometric examination. ${ }^{31}$ In particular, the high rates of autobiographical memory impairment across patients suggest that more routine screening of this domain may provide useful insights into the cognitive integrity and subjective complaints of patients with epilepsy than is typically examined. ${ }^{10,32,33}$

While no laterality effects were evident, verbal ability cannot be assumed to be lateralized to the left hemisphere since it was not directly assessed in these participants (i.e., by fMRI). Another likely limitation of this study was that the verbal memory measure used conflates semantically loaded material with arbitrarily associated material, the latter considered a proximal marker of left mesial temporal lobe disease. ${ }^{3,24}$ As such, a more sensitive measure of verbal arbitrary binding may have disclosed discrete impairments in patients with left HSTLE at a higher rate than in right HS or other TLE types, given their homogeneously located foci. An alternative interpretation is that the lack of laterality effects on neuropsychological functioning in this study is a feature of diffuse, bilateral network abnormalities that can occur in both lesionpositive and -negative TLE. ${ }^{30}$ Future studies should aim to include metrics of wider neuropsychological dysfunction to tease out these potential effects, including measures of language lateralization.

Given that memory and mood disturbances were found to occur in the absence of structural damage, we sought to identify what factors might underpin memory problems in nonlesional disease. Results highlight the multidetermined nature of memory deficits in TLE.

In the first instance, younger age at seizure onset was found to contribute to poor recall of personally relevant memories in MRI-negative TLE, but not lesional TLEs. From a developmental perspective, early seizure onset may disrupt the normal maturation of neural circuits that go on to form a constellation of networks that underpin cognition via physiologic changes such as aberrant sprouting and reduced neurogenesis, ${ }^{34}$ as well as altered functioning and connectivity that stems from the abnormal coactivation of cognitive networks during epileptogenic discharges. ${ }^{35,36}$ Consequently, the cognitive networks of patients with early-onset epilepsy seem to develop abnormally, even in the absence of structural pathology. ${ }^{37}$ Onset of MRI-negative disease in childhood could reasonably accumulate more damage to the function or microscopic integrity of the autobiographical memory network ${ }^{38}$ than adult-onset disease, producing the memory dysfunction evident in our behavioral testing. Alternatively, frequent seizure activity since childhood could interrupt the consolidation of day-to-day events, with "blank spots" or only vague engrams for autobiographical periods surrounding seizures or other subclinical activity. ${ }^{36}$ While neither process would be unique to nonlesional disease, its specificity to MRI-neg TLE in this study may fit with the growing notion that nonlesional epilepsy could be a subtly more diffuse disease process than is seen in circumscribed epileptogenic lesions, for reasons that are yet to be elucidated. ${ }^{39}$

Across both verbal and visual domains of memory, recall impairments in MRI-neg TLE as well as lesional cases were linked to lower general FSIQ. In neurotypical adults, MRI tractography shows that variation in general cognitive ability is related to the integrity of a widely distributed network of white matter involving all cortical lobes of the brain. ${ }^{40}$ This is consistent with findings in epilepsy that (1) HS-TLE and MRI-neg TLE alike show tract-specific atrophy across dispersed brain regions, ${ }^{29}$ and (2) memory deficits in this population are associated with altered integrity of white matter 
tracts. ${ }^{41}$ It is reasonable to speculate that links between lowered FSIQ and recall problems across subtypes of TLE may be the product of subtle, diffuse changes to white matter organization not typically visualized on clinical MRI. A limitation of the current study, however, is the exclusion of patients with intellectual disability. This is an important subgroup of people with epilepsy who require tailored clinical care and specific representation in research, to ensure the generalizability of neuropsychological findings across the epilepsy population. ${ }^{42}$ In addition, the influence of recent ictal electrographic activity on neuropsychological performance could not be reliably evaluated (i.e., time from last seizure to testing) because people with TLE are often amnestic to their seizures and not all patients had seizures captured on video-EEG before testing.

The finding that patients with TLE are prone to mood and memory problems regardless of lesion status implies that different pathophysiologic abnormalities have common effects on behavioral function. We have previously shown that HS-TLE and MRI-neg TLE have distinct network abnormalities, implying that the epileptogenic mechanisms in these subtypes of TLE alter the topography of brain networks in unique ways. ${ }^{30}$ Yet, the current findings suggest that at the group level, the net effect on quantitative neuropsychological performance is indistinguishable. This gives rise to the tantalizing prospect for future investigation that neuropsychological impairments are the emergent property or final common outcome of diverse epileptogenic processes.

\section{Author contributions}

Genevieve Rayner participated sufficiently in the work to take responsibility for content as an author, including drafting/ revising the manuscript for content including medical writing, study concept or design, analysis or interpretation of data, acquisition of data, and statistical analysis. Chris Tailby participated sufficiently in the work to take responsibility for content as an author, including drafting and revising the manuscript for content, study concept, analysis and interpretation of data, statistical analysis, as well as study supervision. Graeme Jackson participated sufficiently in the work to take responsibility for content as an author, including revising the manuscript for content including medical writing, interpretation of data, as well as study supervision. Sarah Wilson participated sufficiently in the work to take responsibility for content as an author, including revising the manuscript for content including medical writing, study concept and design, interpretation of data, as well as study supervision.

\section{Acknowledgment}

The authors thank all the patients and their family members who generously participated in this study for no other reason than to advance neurologic research and improve the quality of life of fellow patients. The authors also gratefully acknowledge the ongoing support of their colleagues at the Florey Institute of Neurosciences and Mental Health, the
Melbourne School of Psychological Sciences at the University of Melbourne, as well as the Comprehensive Epilepsy Programs at the Austin Hospital and Royal Melbourne Hospital. Particular thanks go to Mariana Antoniou for research assistance support, neuropsychologist Dr. David Weintrob for collecting some of the Rey complex figure data as part of his clinical role, Penny Kincade for helping to identify suitable participants, as well as the Director of the Comprehensive Epilepsy Program at the Austin, Prof. Sam Berkovic.

\section{Study funding}

The Article Processing Charge was funded by the authors.

\section{Disclosure}

G. Rayner is in part supported by a National Health and Medical Research Council (NHMRC) project grant (1081151). C. Tailby is in part supported by an NHMRC project grant (1081151). G. Jackson is supported by an NHMRC program grant (1091593), an NHMRC practitioner fellowship (1060312), and by a Victorian Government Operational Infrastructure Support Grant. S. Wilson reports no disclosures relevant to the manuscript. Go to Neurology. org/ $\mathrm{N}$ for full disclosures.

\section{Publication history}

Received by Neurology April 25, 2018. Accepted in final form October 8, 2018 .

\section{References}

1. Nguyen DK, Mbacfou MT, Nguyen DB, et al. Prevalence of nonlesional focal epilepsy in an adult epilepsy clinic. Can J Neurol Sci 2013;40:198-202.

2. Blümcke I, Thom M, Aronica E, et al. International consensus classification of hippocampal sclerosis in temporal lobe epilepsy: a task force report from the ILAE Commission on Diagnostic Methods. Epilepsia 2013;54:1315-1329.

3. Saling MM. Verbal memory in mesial temporal lobe epilepsy: beyond material specificity. Brain 2009;132:570-582.

4. Chelune GJ. Hippocampal adequacy versus functional reserve: predicting memory functions following temporal lobectomy. Arch Clin Neuropsychol 1995;10: 413-432.

5. Helmstaedter C, Petzold I, Bien CG. The cognitive consequence of resecting nonlesional tissues in epilepsy surgery: results from MRI- and histopathology-negative patients with temporal lobe epilepsy. Epilepsia 2011;52:1402-1408.

6. Coras R, Pauli E, Li J, et al. Differential influence of hippocampal subfields to memory formation: insights from patients with temporal lobe epilepsy. Brain 2014;137: $1945-1957$.

7. Hermann B, Loring DW, Wilson S. Paradigm shifts in the neuropsychology of epilepsy. J Int Neuropsychol Soc 2017;23:791-805.

8. Rayner G. The contribution of cognitive networks to depression in epilepsy. Epilepsy Curr 2017;17:78-83.

9. Wilson SJ, Baxendale S. The new approach to classification: rethinking cognition and behavior in epilepsy. Epilepsy Behav 2014;41:307-310.

10. Rayner G, Tailby C. Current concepts of memory disorder in epilepsy: edging towards a network account. Curr Neurol Neurosci Rep 2017;17:55.

11. Scheffer IE, Berkovic S, Capovilla G, et al. ILAE classification of the epilepsies: position paper of the ILAE Commission for Classification and Terminology. Epilepsia 2017;58:512-521.

12. Carne RP, O’Brien TJ, Kilpatrick CJ, et al. MRI-negative PET-positive temporal lobe epilepsy: a distinct surgically remediable syndrome. Brain 2004;127:2276-2285.

13. Giovagnoli AR, Casazza M, Broggi G, et al. Verbal learning and forgetting in patients with temporal lobe epilepsy. Eur J Neurol 2011;3:345-353.

14. Giovagnoli AR, Avanzini G. Learning and memory impairment in patients with temporal lobe epilepsy: relation to the presence, type, and location of brain lesion. Epilepsia 1999;40:904-911.

15. Holdnack J, Drozdick L. Advanced Clinical Solutions (ACS) Test of Premorbid Functioning (TOPF). San Antonio: Pearson Clinical Assessment; 2009.

16. Hurley AD. Mood disorders in intellectual disability. Curr Opin Psychiatry 2006;19. 465.

17. Jackson GD, Berkovic SF, Duncan JS, et al. Optimizing the diagnosis of hippocampal sclerosis using MR imaging. Am J Neuroradiol 1993;14:753-762. 
18. First M, Spitzer R, Gibbon M, et al. Structured Clinical Interview for DSM-IV-TR Axis I Disorders, Research Version, Non-Patient Edition (SCID-I/NP). New York: Biometrics Research, New York Psychiatric Institute; 2002.

19. Blumer D, Montouris G, Davies K. The interictal dysphoric disorder: recognition, pathogenesis, and treatment of the major psychiatric disorder of epilepsy. Epilepsy Behav 2004;5:826-840.

20. Gilliam FG, Barry JJ, Hermann BP, et al. Rapid detection of major depression in epilepsy: a multicentre study. Lancet Neurol 2006;5:399-405.

21. Spitzer RL, Kroenke K, Williams JBW, et al. A brief measure for assessing generalized anxiety disorder: the GAD-7. Arch Intern Med 2006;166:1092-1097.

22. Kopelman M, Wilson B, Baddeley A. The Autobiographical Memory Interview. Bury St. Edmunds: Thames Valley Test Company; 1990.

23. Wechsler D. Wechsler Memory Scale-Fourth Edition. San Antonio: Pearson; 2009.

24. Lillywhite LM, Saling MM, Briellmann RS, et al. Differential contributions of the hippocampus and rhinal cortices to verbal memory in epilepsy. Epilepsy Behav 2007; 10:553-559.

25. Strauss E, Sherman E, Spreen O. A Compendium of Neuropsychological Tests: Administration, Norms, and Commentary, 3rd ed. New York: Oxford University Press; 2006.

26. Benjamini Y, Hochberg Y. Controlling the false discovery rate: a practical and powerful approach to multiple testing. J R Stat Soc Ser B Stat Methodol 1995;57:289-300.

27. Ferrari AJ, Somerville AJ, Baxter AJ, et al. Global variation in the prevalence and incidence of major depressive disorder: a systematic review of the epidemiological literature. Psychol Med 2013;43:471-481.

28. Australian Bureau of Statistics. National Survey of Mental Health and Wellbeing: Summary of Results, 2007. Canberra: Australia Bureau of Statistics; 2008.

29. Vaughan DN, Raffelt D, Curwood E, et al. Tract-specific atrophy in focal epilepsy: disease, genetics, or seizures? Ann Neurol 2017;81:240-250.

30. Vaughan DN, Rayner G, Tailby C, et al. MRI-negative temporal lobe epilepsy: a network disorder of neocortical connectivity. Neurology 2016;87:1934-1942.
31. Wilson SJ, Baxendale S, Barr W, et al. Indications and expectations for neuropsychological assessment in routine epilepsy care: report of the ILAE Neuropsychology Task Force, Diagnostic Methods Commission, 2013-2017. Epilepsia 2015; 56:674-681.

32. Barr WB, Jones JE. A neurodevelopmental approach to cognitive and behavioral assessment in epilepsy. Neurology 2016;87:1634-1635.

33. Rayner G, Jackson GD, Wilson SJ. Mechanisms of memory impairment in epilepsy depend on age at disease onset. Neurology 2016;87:1642-1649.

34. Ben-Ari Y, Holmes GL. Effects of seizures on developmental processes in the immature brain. Lancet Neurol 2006;5:1055-1063.

35. Pillay N, Archer JS, Badawy RAB, et al. Networks underlying paroxysmal fast activity and slow spike and wave in Lennox-Gastaut syndrome. Neurology 2013;81:665-673.

36. Dinkelacker V, Xin X, Baulac M, et al. Interictal epileptic discharge correlates with global and frontal cognitive dysfunction in temporal lobe epilepsy. Epilepsy Behav 2016;62:197-203.

37. Curwood EK, Pedersen M, Carney PW, et al. Abnormal cortical thickness connectivity persists in childhood absence epilepsy. Ann Clin Transl Neurol 2015;2: 456-464.

38. Tailby C, Rayner G, Wilson S, et al. The spatiotemporal substrates of autobiographical recollection: using event-related ICA to study cognitive networks in action. Neuroimage 2017;152:237-248.

39. So E, Ryvlin P, editors. MRI-Negative Epilepsy: Evaluation and Surgical Management. Cambridge, UK: Cambridge University Press; 2015.

40. Malpas CB, Genc S, Saling MM, et al. MRI correlates of general intelligence in neurotypical adults. J Clin Neurosci 2016;24:128-134.

41. Riley JD, Franklin DL, Choi V, et al. Altered white matter integrity in temporal lobe epilepsy: association with cognitive and clinical profiles. Epilepsia 2010;51:536-545.

42. Kerr M, Linehan C, Brandt C, et al. Behavioral disorder in people with an intellectual disability and epilepsy: a report of the Intellectual Disability Task Force of the Neuropsychiatric Commission of ILAE. Epilepsia Open 2016;1:102-111. 


\section{Neurology}

\section{Looking beyond lesions for causes of neuropsychological impairment in epilepsy}

Genevieve Rayner, Chris Tailby, Graeme Jackson, et al.

Neurology 2019;92;e680-e689 Published Online before print January 11, 2019

DOI 10.1212/WNL.0000000000006905

\section{This information is current as of January 11, 2019}

Updated Information \& Services

References

Permissions \& Licensing

Reprints including high resolution figures, can be found at: http://n.neurology.org/content/92/7/e680.full

This article cites 35 articles, 4 of which you can access for free at: http://n.neurology.org/content/92/7/e680.full\#ref-list-1

Information about reproducing this article in parts (figures,tables) or in its entirety can be found online at:

http://www.neurology.org/about/about_the_journal\#permissions

Information about ordering reprints can be found online:

http://n.neurology.org/subscribers/advertise

Neurology ${ }^{\circledR}$ is the official journal of the American Academy of Neurology. Published continuously since 1951, it is now a weekly with 48 issues per year. Copyright Copyright ( 2019 The Author(s). Published by Wolters Kluwer Health, Inc. on behalf of the American Academy of Neurology.. All rights reserved. Print ISSN: 0028-3878. Online ISSN: 1526-632X.

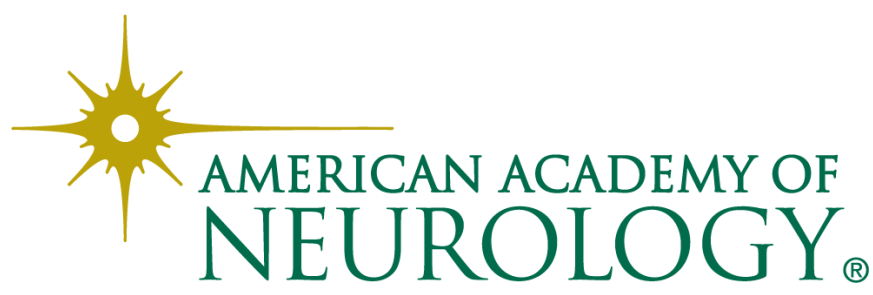

